INPLASY

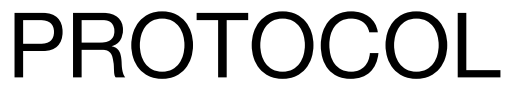

To cite: Yang et al.

Effectiveness of Acupuncture in the Treatment of

Neurodermatitis: A Systematic

Review and Meta-Analysis.

Inplasy protocol 2021110041.

doi:

10.37766/inplasy2021.11.0041

Received: 13 November 2021

Published: 13 November 2021

Corresponding author:

Yu Lai

archimedean@rocketmail.com

Author Affiliation:

Chengdu University of

Traditional Chinese Medicine.

Support: Yes.

Review Stage at time of this submission: Preliminary

searches.

Conflicts of interest:

None declared.

\section{Effectiveness of Acupuncture in the Treatment of Neurodermatitis: A Systematic Review and Meta-Analysis}

Yang, L1; Li, XY2; Huang, W3; Li, JL4; Lai, Y5.

Review question / Objective: Effectiveness of acupuncture in the treatment of neurodermatitis: a systematic review and Meta-Analysis.

Condition being studied: Neurodermatitis is a skin disease with severe itching and recurring episodes. This study explores the clinical efficacy of acupuncture and moxibustion in the treatment of neurodermatitisNeurodermatitis is a severe itching and recurring skin disease.

Eligibility criteria: Inclusion criteria: (1) Participants: The subject is a clinically diagnosed neurodermatitis patient. There are no restrictions on language, age, gender, country, or race. (2) Research type: randomized controlled experiment. (3) Intervention: mainly acupuncture and moxibustion. And compared with the control group. (4) Control group: with drug treatment or no treatment. (5) Results: No itching, the symptoms disappeared, and the skin lesions returned to normal. Exclusion criteria: (1) Other traditional Chinese methods. (2) Exclude duplicate publications and research with incomplete data. (3) Use acupuncture plus other drugs. (4) Discussion on the exclusion theory.

INPLASY registration number: This protocol was registered with the International Platform of Registered Systematic Review and Meta-Analysis Protocols (INPLASY) on 13 November 2021 and was last updated on 13 November 2021 (registration number INPLASY2021110041).

\title{
INTRODUCTION
}

Review question / Objective: Effectiveness of acupuncture in the treatment of neurodermatitis: a systematic review and Meta-Analysis
Condition being studied: Neurodermatitis is a skin disease with severe itching and recurring episodes. This study explores the clinical efficacy of acupuncture and moxibustion in the treatment of 
neurodermatitisNeurodermatitis is a severe itching and recurring skin disease.

\section{METHODS}

Participant or population: Neurodermatitis.

Intervention: Acupuncture.

Comparator: Other treatments.

Study designs to be included: Randomized clinical trials (RCTs).

Eligibility criteria: Inclusion criteria: (1) Participants: The subject is a clinically diagnosed neurodermatitis patient. There are no restrictions on language, age, gender, country, or race. (2) Research type: randomized controlled experiment. (3) Intervention: mainly acupuncture and moxibustion. And compared with the control group. (4) Control group: with drug treatment or no treatment. (5) Results: No itching, the symptoms disappeared, and the skin lesions returned to normal. Exclusion criteria: (1) Other traditional Chinese methods. (2) Exclude duplicate publications and research with incomplete data. (3) Use acupuncture plus other drugs. (4) Discussion on the exclusion theory.

Information sources: $\mathbf{A}$ total of $\mathbf{8}$ databases were searched including PubMed, Cochrane Library, EMBASE, Web of Science, China Knowledge Infrastructure (CNKI), China Biomedicine (CBM), Wanfang Database, and China Science and Chinese Science Journal Database (VIP).

Main outcome(s): Evaluation of the safety and effectiveness of acupuncture and moxibustion in the treatment of neurodermatitis.

Quality assessment / Risk of bias analysis: Two authors independently included and excluded the literature. Use Review Manager 5.4 software to screen the literature, extract data and evaluate the quality of the literature.

Strategy of data synthesis: Use RevMan 5.4 software downloaded from the Cochrane website to analyze the data included in the literature.

Subgroup analysis: Medicine and acupunctureSubgroup analysis was performed where necessary.

Sensitivity analysis: Sensitivity analysis was performed where necessary.

Country(ies) involved: China.

Keywords: Neurodermatitis; Acupuncture; Systematic Review; Meta-Analysis.

Contributions of each author:

Author 1 - Lin Yang.

Email: 781387628@qq.com

Author 2 - Xinyun Li.

Email: 1043541625@qq.com

Author 3 - Wei Huang.

Email: gracehw@126.com

Author 4 - Jialiang Li.

Email: 303738554@qq.com

Author 5 - Yu Lai.

Email: archimedean@rocketmail.com 